\title{
Tráfico de partes e individuos del oso andino Tremarctos ornatus en el Perú
}

\author{
Judith Figueroa \\ Grupo de Investigación de Zoología de Vertebrados, Universidad de Alicante, España \\ Asociación para la Investigación y Conservación de la Biodiversidad (AICB), Lima, Perú
}

\begin{abstract}
Resumen
Entre el 2002 y el 2007 se registró la venta de partes y productos derivados del oso andino Tremarctos ornatus en 27 de los 45 mercados, ubicados en 14 de las 24 regiones visitadas en el Perú. Las regiones que presentaron mayor volumen de ventas de partes fueron Lambayeque (32,7 \%), Piura (17,3\%), San Martín (13,5 \%), Cajamarca $(11,5 \%)$ y Amazonas (5,8 \%). Los principales productos a la venta fueron la llamada "frotación de oso" (grasa de oso mezclada con hierbas) $(38,4 \%)$, la grasa $(17,4 \%)$, las patas $(14 \%)$, la piel $(10,5 \%)$ y los huesos $(8,1 \%)$. Las partes procedían principalmente de osos cazados en Amazonas (27,6 \%), Lambayeque (20,7 \%) y San Martín $(13,8 \%)$. La venta de partes de oso se hace localmente para usos curativos, mágicos, afrodisíacos y alimenticios, aunque también se registró la venta de vesícula biliar a personas de origen asiático en Amazonas y Cusco; en esta última región, el valor de este producto fue de US\$ 580. Se registró, igualmente, la venta de oseznos a precios entre US\$29 y 1.000 , dependiendo del lugar de la venta.
\end{abstract}

Palabras clave: CITES, fauna silvestre, oso andino, Perú, tráfico, Tremarctos ornatus.

Traffic of parts and individuals of Andean bear Tremarctos ornatus in Perú

\begin{abstract}
Between 2002 and 2007, the sale of parts and derivatives of the Andean bear Tremarctos ornatus was registered in 27 of the 45 markets located in 14 of 24 regions visited in Peru. The regions with the highest sales of bear parts were Lambayeque (32.7\%), Piura (17.3\%), San Martín (13.5\%), Cajamarca (11.5\%) and Amazonas (5.8 \%). The main products on sale were bear fat cream (38.4\%), fat (17.4\%), paws (14\%), leather $(10.5 \%)$ and bones $(8.1 \%)$. The parts came mainly from bears hunted in Amazonas (27.6\%), Lambayeque (20.7 \%) and San Martin (13.8 \%). Bear parts are sold locally mainly for magical and curative uses, although they are also offered as aphrodisiac or food. However, the sale of gallbladder to Asians was registered in Amazonas and Cusco, location where the price was US\$ 580. Bear cubs prices fluctuated between US\$ 29 and 1,000, depending on the area.
\end{abstract}

Key words: Andean bear, CITES, Perú, traffic, wildlife, Tremarctos ornatus.

\section{Introducción}

El tráfico ilegal de vida silvestre es una de las actividades criminales más lucrativas del mundo, tanto que ocupa el quinto lugar entre estas y solo lo supera el tráfico de drogas, el de personas, el de petróleo y la falsificación, con ingresos entre US\$ 7 y 10 mil millones al año (IFAW, 2013). El Perú cuenta con 11 ecorregiones conformadas por 84 zonas de vida de las 117 reconocidas en el mundo y con 28 de los 32 tipos de clima, lo que determina su gran biodiversidad. Hasta el momento, se han identificado 508 especies de mamíferos, 1.835 de aves, 421 de reptiles, 538 de anfibios y 1.064 de peces de aguas continentales, ubicando al Perú como el tercer país con mayor diversidad de especies animales en América y el quinto en el mundo (Villena
Arboccó, 2013). Además, el país cuenta con más de 4.400 especies de plantas nativas con propiedades conocidas y 128 especies nativas domesticadas, ocupando el primer lugar en el mundo en estas categorías. En su territorio también hay 4.000 especies de orquídeas y 600 especies forestales y concentra $7,8 \%$ de las plantas cultivables y $10 \%$ de las especies de flora del planeta. Asimismo, ocupa el noveno lugar en endemismo de flora y fauna silvestre (MINAG, 2013).

\section{Correspondencia:}

Judith Figueroa, aicb.peru@gmail.com

Recibido: 21 de octubre de 2013

Aceptado: 10 de mayo de 2014 
Como lo señalan Mancera Rodríguez \& Reyes García (2008) con relación a Colombia, esta gran biodiversidad ha convertido al Perú en un centro de comercio ilegal de vida silvestre y, pese a la legislación existente y a las medidas adoptadas hasta ahora para fomentar su uso sostenible y garantizar su protección, se presume que el volumen del tráfico ilegal sigue siendo de gran magnitud. Debido a la naturaleza ilícita de esta actividad, así como a las pocas cifras disponibles sobre extracción y comercialización de fauna en el país, y al mal uso que las autoridades ambientales dan a sus recursos económicos, se carece de parámetros que permitan establecer, cualitativa o cuantitativamente, el impacto biológico en cada especie, así como el impacto sobre los ecosistemas.

En el caso específico del oso andino Tremarctos ornatus hay leyes y normas que lo protegen. A nivel internacional, T. ornatus está incluido en el Apéndice I de la Convención sobre el Comercio Internacional de Especies Amenazadas de Fauna y Flora Silvestres como especie en peligro de extinción, que está o puede verse afectada por el comercio de todas sus partes y derivados (CITES, 2013). Además, la Unión Internacional para la Conservación de la Naturaleza lo ha clasificado como especie en situación vulnerable (IUCN, 2013). En el Perú el oso comenzó a protegerse legalmente desde la década de 1970, específicamente mediante la Resolución Ministerial No 5056-70-AG de 1970, que vedó indefinidamente su caza; mediante el Decreto Ley $\mathrm{N}^{\circ}$ 21080 de 1975 se le incorporó a la CITES; en la Resolución Ministerial No 1710-77-AG de 1977 y en la No $01082-$ 90-AG de 1990, se le categorizó en situación vulnerable (Pulido, 1991); posteriormente, en el Decreto Supremo N ${ }^{\circ}$ 013-99-AG de 1999 se le clasificó como especie en vías de extinción y en el $\mathrm{N}^{\circ}$ 034-2004-AG de 2004, como especie en peligro, prohibiéndose su caza, captura, tenencia, transporte o exportación con fines comerciales (MINAG, 2004). El artículo 176 del Reglamento de la Ley Forestal y de Fauna Silvestre (Ley $\mathrm{N}^{\circ} 27308$ ) de 2001, promulgado mediante el Decreto Supremo No 014-2001-AG, y el artículo 94 de la Ley Forestal y de Fauna Silvestre No 29763 de 2011, señalan que no puede autorizarse la cría de las especies incluidas en el Apéndice I de la CITES y de otras especies amenazadas en zoocriaderos, que son establecimientos para el manejo ex situ de fauna silvestre con fines comerciales y para la producción de bienes y servicios (Congreso de la República, 2011).

A pesar de esta normativa legal, la venta de las partes del oso se registra en todos los países donde este se distribuye: Venezuela (Herrera, et al., 1994), Colombia (Orejuela \& Jorgenson, 1996), Ecuador (Peyton, et al., 1998; WSPA, 2002a), Perú (Figueroa \& Stucchi, 2002; Figueroa, 2003; Amanzo, et al., 2007a, 2007b; Figueroa, et al., 2013) y Bolivia (Salazar \& Anderson, 1990; Paisley, 2001). Sin embargo, se desconoce el alcance de este comercio. El presente estudio tuvo el objetivo de determinar quiénes, dónde, cómo y qué motiva el comercio de partes y de individuos de oso andino en los principales mercados del Perú.

\section{Metodología}

Entre febrero de 2002 y noviembre de 2007 se visitaron y entrevistaron a vendedores de 45 mercados en 24 regiones del Perú (Figura 1, Tabla 1). Se hicieron las siguientes preguntas sobre las partes del oso: ¿cuál es su costo?, ¿se puede hacer algún pedido específico?, y ¿de dónde provienen? Además, se preguntó específicamente la edad de los individuos.

Para una mejor caracterización, las partes observadas se clasificaron de la siguiente manera: hueso peneano: este se vende de forma independiente debido a su demanda como afrodisíaco para los hombres; otros huesos: partes de huesos o estructura completa del omóplato, del radio, del húmero, del fémur, etc.; grasa de oso: de consistencia compacta y color beige, se le llama manteca o sebo, y se vende como aceite después de derretirla; garra: uña, parte dura, de naturaleza córnea, que crece en las extremidades de los dedos del oso; pata: pie, parte terminal del miembro posterior o anterior; "frotación de oso": según se indica en el producto, es una mezcla de grasa de oso andino y hierbas medicinales a la que se le atribuyen propiedades curativas.

\section{Resultados}

La venta de partes del oso andino y de frotación de oso se encontró en 27 (60\%) mercados de 14 regiones del Perú. En Lima se registró el 42,4 \% de la venta de la frotación de oso y el 3,8 \% de la venta de partes de oso. Por el contrario, en Lambayeque, el 32,7 \% correspondió a la venta de partes de oso y el 3,0 \% a la de frotación de oso (Tabla 2). En Chiclayo (Lambayeque), se encontró la mayor cantidad y variedad de partes del oso andino en venta en el Perú, que se renuevan constantemente según se constató en las seis visitas realizadas. De acuerdo a uno de los vendedores, esto se debe a la demanda de los curanderos y de los habitantes de la zona, quienes las usan con fines medicinales. Además de Lambayeque, los mercados con mayor venta de partes de oso también se ubicaron en el norte: Piura, San Martín, Cajamarca y Amazonas, con 17,3, 13,5, 11,5 y 5,8\%, respectivamente.

Las partes procedían principalmente de osos cazados en Amazonas (27,6 \%), Lambayeque (20,7 \%) y San Martín $(13,8 \%)$ (Tabla 3). En Amazonas, los lugares específicos de procedencia fueron Santa María de Nieva, Chiriaco, Imacita, Quijalca, Valle de los Chilchos y Lajasbamba; en Ayacucho, San Francisco; en Cajamarca, las zonas limítrofes con Amazonas; en Cusco, Quillabamba; en Huánuco, Carpish y Divisoria Padre Abad; en Junín, Satipo, San Ramón y La Merced; en Lambayeque, Salas, Chongoyape, Oyotún, Colán y Pampa Grande; en Piura, Huancabamba y Ayabaca; en Puno, 


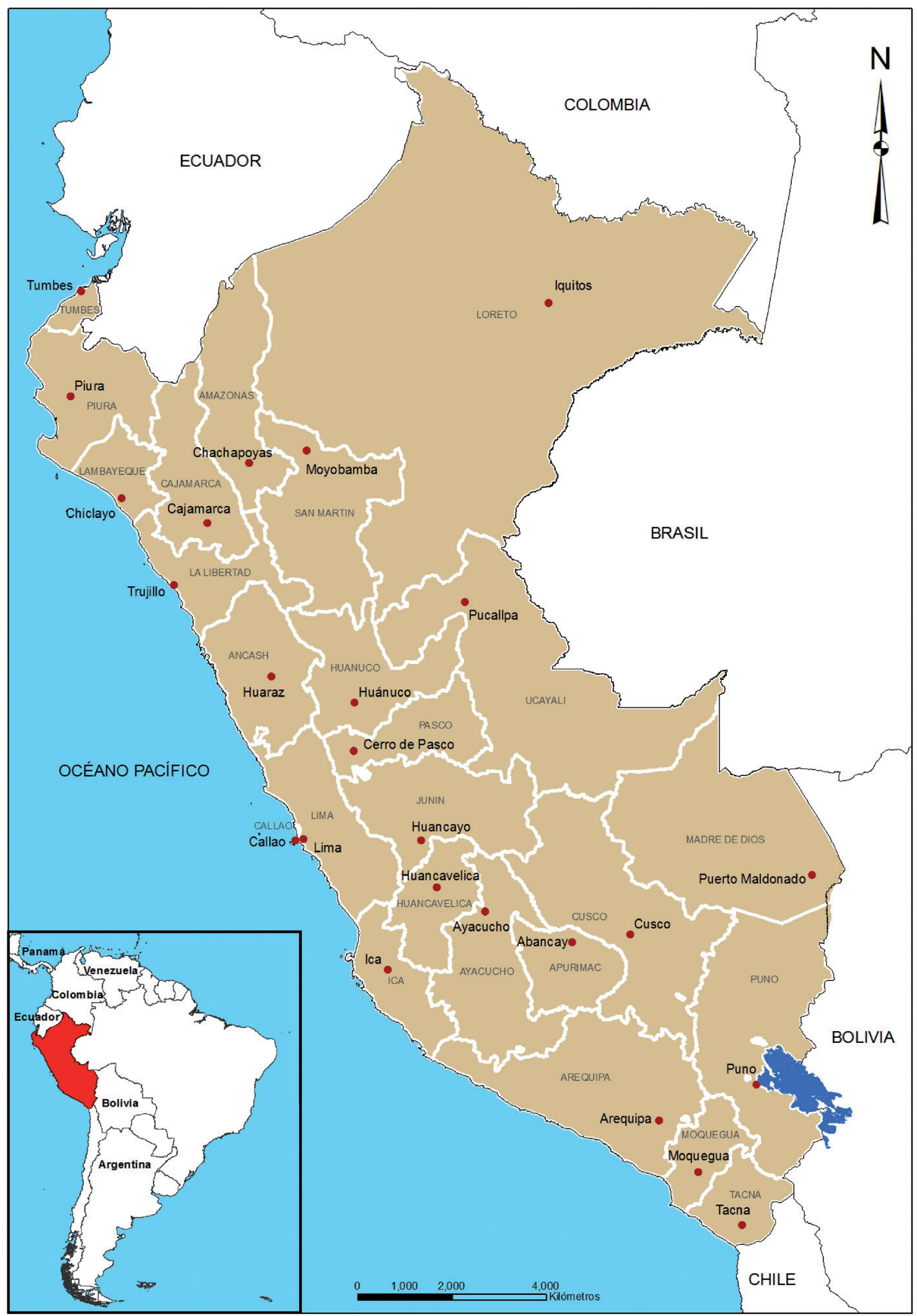

Figura 1. Ubicación de las regiones visitadas en el Perú 
Tabla 1. Mercados visitados en el Perú entre el 2001 y el 2007

\begin{tabular}{|c|c|c|c|c|c|c|}
\hline $\mathbf{N}^{\mathbf{o}}$ & Región & Localidades & Año & $\begin{array}{c}\text { Mercados } \\
\text { visitados }\end{array}$ & $\begin{array}{c}\text { Venta de partes } \\
\text { de oso } \\
\end{array}$ & $\begin{array}{c}\text { Venta de frotación } \\
\text { de oso }\end{array}$ \\
\hline 1 & Amazonas & Chachapoyas & 2002 & 2 & 2 & 1 \\
\hline 2 & Ancash & Huaraz & 2002 & 2 & 0 & 2 \\
\hline 3 & Apurímac & Abancay & 2002 & 1 & 0 & 0 \\
\hline 4 & Arequipa & Arequipa & 2003 & 1 & 1 & 1 \\
\hline 5 & Ayacucho & San Miguel & 2005 & 1 & 1 & 0 \\
\hline 6 & Cajamarca & Cajamarca, Cutervo & 2002 & 2 & 2 & 0 \\
\hline 7 & Cusco & Cusco, Pisaq & 2001,2003 & 2 & 2 & 1 \\
\hline 8 & Huancavelica & Huancavelica & 2007 & 1 & 0 & 0 \\
\hline 9 & Huánuco & Huánuco, Tingo María & 2002,2007 & 4 & 0 & 3 \\
\hline 10 & Ica & Ica & 2005 & 1 & 0 & 0 \\
\hline 11 & Junín & Huancayo & 2003, 2007 & 1 & 1 & 1 \\
\hline 12 & La Libertad & Trujillo & 2002 & 2 & 1 & 1 \\
\hline 13 & Lambayeque & Chiclayo, Oyotún, Motupe & 2002,2003 & 4 & 4 & 0 \\
\hline 14 & Lima & Lima & 2007 & 1 & 1 & 1 \\
\hline 15 & Loreto & Iquitos & 2005 & 1 & 0 & 0 \\
\hline 16 & Madre de Dios & Puerto Maldonado & 2003 & 1 & 0 & 0 \\
\hline 17 & Moquegua & Moquegua & 2003 & 1 & 0 & 0 \\
\hline 18 & Pasco & Oxapampa, Villa Rica, Cerro de Pasco & $2003,2005,2007$ & 4 & 0 & 0 \\
\hline 19 & Piura & Morropón, Sullana & 2007 & 3 & 3 & 1 \\
\hline 20 & Puno & Puno, Juliaca, Sandia & 2006 & 3 & 0 & 0 \\
\hline 21 & San Martín & Tarapoto, Moyobamba, Nueva Cajamarca & 2002 & 3 & 3 & 0 \\
\hline 22 & Tacna & Tacna & 2003 & 1 & 0 & 0 \\
\hline 23 & Tumbes & Tumbes & 2004 & 1 & 0 & 0 \\
\hline 24 & Ucayali & Pucallpa & 2002,2007 & 2 & 1 & 0 \\
\hline
\end{tabular}

Tabla 2. Mercados con venta de partes y frotación de oso andino

\begin{tabular}{lccllcc}
\hline \multirow{2}{*}{ Mercado } & \multicolumn{2}{c}{ Venta (\%) } & & \multicolumn{2}{c}{ Venta (\%) } \\
\cline { 2 - 3 } \cline { 6 - 6 } & $\begin{array}{c}\text { Parte } \\
\text { de oso }\end{array}$ & $\begin{array}{c}\text { Frotación } \\
\text { de oso }\end{array}$ & Mercado & $\begin{array}{r}\text { Parte } \\
\text { de oso }\end{array}$ & $\begin{array}{c}\text { Frotación } \\
\text { de oso }\end{array}$ \\
\hline Amazonas & 5,8 & 3,0 & Junín & 1,9 & 9,1 \\
\hline Ancash & -- & 6,1 & La Libertad & 1,9 & 3,0 \\
\hline Arequipa & 1,9 & 9,1 & Lambayeque & 32,7 & 3,0 \\
\hline Ayacucho & 3,8 & -- & Lima & 3,8 & 42,4 \\
\hline Cajamarca & 11,5 & 6,1 & Piura & 17,3 & 6,1 \\
\hline Cusco & 1,9 & 6,1 & San Martín & 13,5 & -- \\
\hline Huánuco & 1,9 & 6,1 & Ucayali & 1,9 & -- \\
\hline Total & & & & 61,2 & 38,8 \\
\hline
\end{tabular}

Sandia, San Gabán, Patambuco y Putina, y en San Martín, las áreas colindantes con el Bosque de Protección Alto Mayo. Juzgando por el número de pieles a la venta, se debieron cazar al menos 20 osos para el comercio de sus partes.

La frotación de oso fue el producto más comúnmente encontrado en los mercados visitados $(38,4 \%)$ (Figura 1S, http://www.raccefyn.co/index.php/raccefyn/article/
downloadSuppFile/62/90), seguido de la grasa $(17,4 \%)$ (Figura 2S, http://www.raccefyn.co/index.php/raccefyn/ article/downloadSuppFile/62/91), las patas (14\%) (Figura 3S, http://www.raccefyn.co/index.php/raccefyn/article/ downloadSuppFile/62/92), la piel (10,5\%), los huesos $(8,1 \%)$ y el hueso peneano $(4,7 \%$ ) (Figura $4 \mathrm{~S}$, http://www.raccefyn. co/index.php/raccefyn/article/downloadSuppFile/62/93) (Tabla 4). A pesar de que los empaques de la frotación de oso presentaban en la etiqueta imágenes del oso polar Ursus maritimus, del oso pardo Ursus arctos, del oso negro americano Ursus americanus y del panda Ailuropoda melanoleuca, los vendedores aseguraron que la mezcla estaba hecha con la grasa del oso andino.

En los mercados visitados, $10 \mathrm{ml}$ de aceite costaban entre US\$ 0,4 y 3,0. Cada garra se ofrecía a un precio entre US\$ 1,5 y 14,5 , dependiendo del tamaño, y la pata completa, entre US\$ 4,3 y 17,4. La carne de oso solo se ofrecía en venta en los mercados de Chiclayo, Chachapoyas (Amazonas) y Sullana (Piura), a un precio entre US\$ 8,5 a 10,0 , aunque debía solicitarse con días o semanas de anticipación y asegurar su compra por medio de un adelanto. También en la feria de Oyotún (Lambayeque) un cazador reportó la 
Tabla 3. Procedencia de las partes del oso andino que se encontraron a la venta

\begin{tabular}{|c|c|c|c|c|c|c|c|c|c|c|}
\hline \multirow{2}{*}{ Mercado } & \multicolumn{10}{|c|}{ Procedencia de las partes del oso (\%) } \\
\hline & Amazonas & Ayacucho & Cajamarca & Cusco & Huánuco & Junín & Lambayeque & Piura & Puno & San Martín \\
\hline Amazonas & 100 & & & & & & & & & \\
\hline Arequipa & & & & & & & & & 100 & \\
\hline Ayacucho & & 100 & & & & & & & & \\
\hline Cajamarca & 50 & & 50 & & & & & & & \\
\hline Cusco & & & & 100 & & & & & & \\
\hline Huánuco & & & & & 100 & & & & & \\
\hline Junín & & & & & & 100 & & & & \\
\hline La Libertad & 50 & & & & & & 50 & & & \\
\hline Lambayeque & 16,7 & & 16,7 & & & & 66,7 & & & \\
\hline Lima & & & & & & & 50 & & 50 & \\
\hline Piura & 33,3 & & & & & & & 33,3 & & 33,3 \\
\hline San Martín & 33,3 & & & & & & & & & 66,7 \\
\hline Ucayali & & & & & 100 & & & & & \\
\hline Total & 27,6 & 3,4 & 6,9 & 3,4 & 6,9 & 3,4 & 20,7 & 6,9 & 6,9 & 13,8 \\
\hline
\end{tabular}

Tabla 4. Partes de oso y derivados a la venta en los mercados visitados

\begin{tabular}{|c|c|c|c|c|c|c|c|c|c|c|}
\hline \multirow{2}{*}{ Mercado } & \multicolumn{10}{|c|}{ Partes del oso andino que se venden en los mercados visitados (\%) } \\
\hline & Grasa & Frotación de oso & Sangre & $\begin{array}{c}\text { Hueso } \\
\text { peneano }\end{array}$ & $\begin{array}{c}\text { Otros } \\
\text { huesos }\end{array}$ & Cráneo & Pata y garra & $\begin{array}{c}\text { Vesícula } \\
\text { biliar }\end{array}$ & Piel & Carne \\
\hline Amazonas & 40 & 20 & & & & & & & 40 & \\
\hline Ancash & & 100 & & & & & & & & \\
\hline Arequipa & 25 & 75 & & & & & & & & \\
\hline Ayacucho & 50 & & & & & & & & 50 & \\
\hline Cajamarca & 25 & 25 & & 12,5 & 12,5 & & 12,5 & & 12,5 & \\
\hline Cusco & & 66,7 & & & & & 33,3 & & & \\
\hline Huánuco & & 66,7 & & & & & & & 33,3 & \\
\hline Junín & 25 & 75 & & & & & & & & \\
\hline La Libertad & & 50 & & & & & 50 & & & \\
\hline Lambayeque & 11,1 & 5,6 & & 11,1 & 22,2 & 5,6 & 22,2 & 5,6 & 5,6 & 11,1 \\
\hline Lima & 6,3 & 87,5 & & & & & 6,3 & & & \\
\hline Piura & 36,4 & 18,2 & & & 9,1 & & 18,2 & & 9,1 & 9,1 \\
\hline San Martín & 14,3 & & & 14,3 & 14,3 & & 28,6 & & 28,6 & \\
\hline Ucayali & & & 100 & & & & & & & \\
\hline Total & 17,4 & 38,4 & 1,2 & 4,7 & 8,1 & 1,2 & 14 & 1,2 & 10,5 & 3,5 \\
\hline
\end{tabular}

venta eventual de la carne de oso a US\$ 1,7 por kilo. Solo se encontró la venta de una vesícula biliar deshidratada en el mercado de Chiclayo, cuyo precio era de US\$ 5,8 (Figura 5S, http://www.raccefyn.co/index.php/raccefyn/article/ downloadSuppFile/62/94) (Tabla 5); sin embargo, se obtuvo información de dos casos de venta de hígado y vesícula a personas de origen asiático, en Amazonas y Cusco. En este último lugar, el comprador pidió la oreja del oso para comprobar su origen; el costo fue de US\$ 580.
En Challabamba (Cusco) se registró la venta de dos pieles de osos cazados en la margen izquierda del río Yavero, en la zona de amortiguamiento del Parque Nacional del Manu; las pieles estaban siendo ofrecidas directamente por los cazadores a US\$ 29 en el poblado, a US\$ 34,8 en el mercado de Sicuani y a US\$ 100 en el mercado de Cusco. En Ayabaca (Piura), se encontró una piel de oso cazado en Yanta que iba a ser comercializada (Alejandro Zegarra, comunicación personal, 2006). Asimismo, se tiene el reporte de que una 
Tabla 5. Precio de venta (US\$) de partes y frotación de oso andino

\begin{tabular}{|c|c|c|c|c|c|c|c|c|c|c|c|c|c|}
\hline \multirow{2}{*}{ Mercado } & \multicolumn{8}{|c|}{ Grasa (ml) } & \multicolumn{4}{|c|}{ Frotación de oso (g) } & \multirow{2}{*}{$\begin{array}{c}\text { Sangre }(\mathrm{ml}) \\
180\end{array}$} \\
\hline & 5 & 10 & 20 & 30 & 50 & 100 & 250 & 1000 & 15 & 20 & 28 & 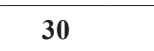 & \\
\hline Amazonas & 1,5 & & & & & & & & & 1,5 & & & \\
\hline Ancash & & & & & & & & & $9-1,16$ & & & & \\
\hline Arequipa & 0,9 & & & & & & & & 0,6 & & & 1,16 & \\
\hline Ayacucho & & & & & & & 1,16 & & & & & & \\
\hline Cajamarca & 1,5 & & 0,9 & & & & & & 0,6 & 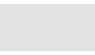 & . & 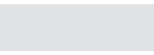 & \\
\hline Cusco & & & & & & & & & $0,7-0,9$ & & & & \\
\hline Huánuco & & & & & & & & & 0,7 & 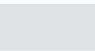 & 1,5 & & \\
\hline Junín & 0,58 & & & & & & & & 0,3 & & & & \\
\hline La Libertad & & & & & & & & & & 0,6 & 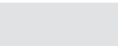 & & \\
\hline Lambayeque & 0,58 & & 0,9 & & & 5,8 & & & & & & & \\
\hline Lima & & & &, 5 & & & & & $0,15-0,3$ & & 0,7 & & \\
\hline Piura & & 1,7 & &, 7 & 7 & & & 5 & 0,7 & 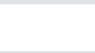 & 1,5 & & \\
\hline San Martín & & & & ,9 & & & & & & & & & \\
\hline Ucayali & & & & & & & & & & & & & 14,5 \\
\hline \multirow{2}{*}{ Mercado } & \multicolumn{7}{|c|}{ Hueso } & \multirow{2}{*}{\multicolumn{2}{|c|}{ Pata }} & \multirow{2}{*}{ Garra } & \multirow{2}{*}{$\begin{array}{l}\text { Vesícula } \\
\text { biliar }\end{array}$} & \multirow{2}{*}{ Piel } & \multirow{2}{*}{$\begin{array}{c}\text { Carne } \\
\text { (kg) }\end{array}$} \\
\hline & Fémur & Omóplato & Cráneo & Carpo & Peneano & Radio & Otros & & & & & & \\
\hline Amazonas & & & & & & & & & & & & 29 & \\
\hline \multicolumn{14}{|l|}{ Ancash } \\
\hline \multicolumn{14}{|l|}{ Arequipa } \\
\hline Ayacucho & & & & & & & & & & & & 29 & \\
\hline Cajamarca & & & & & 1,5 & & 1,5 & & 7 & & & $4,3\left(25 \mathrm{~cm}^{2}\right)$ & \\
\hline Cusco & & & & & & & & & 9 & 14,5 & & & \\
\hline Huánuco & & & & & & & & & & & & $29-43,5$ & \\
\hline \multicolumn{14}{|l|}{ Junín } \\
\hline La Libertad & & & & & & & & & ,4 & & & & \\
\hline Lambayeque & 11,6 & 7,25 & 23,2 & 5,8 & 14,5 & & & $5,8-1$ & $4 * 72,5$ & & 5,8 & 34,8 & $1,7-10$ \\
\hline \multicolumn{14}{|l|}{ Lima } \\
\hline Piura & & & & & & 1,7 & & & & 7 & & 70 & $8,5-10$ \\
\hline San Martín & & & & & 0,3 & & 2,9 & & -29 & 1,5 & & $23,2-43,5$ & \\
\hline Ucayali & & & & & & & & & & & & & \\
\hline
\end{tabular}

*Estructura conformada por pata de oso y espada de bronce usada por los curanderos de la zona norte en sus ritos.

persona llevó al mercado de Juliaca (Puno) una piel de oso cazado en Sandia (Puno) para ofrecerla al público, pero nadie se interesó en comprarla por su alto precio y porque no le encontraron utilidad (Trinidad Tapia, comunicación personal, 2007).

También se observó la venta de partes de otros ejemplares de fauna en peligro. En Pisaq (Cusco) se encontraron patas y plumas del cóndor andino Vultur gryphus a US\$ 34,8 y US\$ 5,8 , respectivamente, y una piel de pingüino de Humboldt Spheniscus humboldti a US\$ 2,9. En Tingo María (Huánuco) se vendían pieles del oso hormiguero gigante Myrmecophaga tridactyla a US\$ 30; en Trujillo
(La Libertad), uñas del tapir amazónico Tapirus terrestris, cada una a US\$ 5; en Chiclayo (Lambayeque), se registró el ofrecimiento de la mitad del cuerpo de un cóndor andino a US\$ 72, de un gallito de las rocas Rupicola peruviana a US\$ 20 (Figura 6S, http://www.raccefyn.co/index.php/ raccefyn/article/downloadSuppFile/62/95), de las patas del tapir de páramo Tapirus pinchaque a US\$ 14,5 (Figura 7S, http://www.raccefyn.co/index.php/raccefyn/article/ downloadSuppFile/62/96) y de la piel de un puma Puma concolor. En Tarapoto y Nueva Cajamarca (San Martín), se encontraron pieles de jaguar Panthera onca y de puma, y patas de tapir amazónico. En Pucallpa (Ucayali), se 
registró la venta de pieles de tapir amazónico, de jaguar, de puma, de oso hormiguero gigante, de huangana Tayassu pecari, de nutria de río Lontra longicaudis, de lobo de río Pteronura brasiliensis y de pacarana Dinomys branickii (Figura 8S, http://www.raccefyn.co/index.php/raccefyn/ article/downloadSuppFile/62/97).

Aunque solo se reportó el caso de un osezno vendido en el mercado de Mochoqueque (Lambayeque) en el año 2001, se obtuvieron registros de ventas directas por parte de los cazadores, principalmente de oseznos menores de un año de edad, en las comunidades de Achamal y La Jalca (Amazonas) en el 2001 y el 2002; en Mochumí (Lambayeque), donde se encontró una osezna mantenida en condiciones precarias que iba a ser vendida en Chiclayo a US\$ 500 (Figura 9S, http://www.raccefyn.co/index.php/raccefyn/article/ downloadSuppFile/62/98); en Mal Paso (Pasco), en donde en el 2003 un poblador propuso la venta de dos oseznos de cuatro meses provenientes de Quitasol-Layahuas a US\$ 29 cada uno (de no lograrse la venta, estos serían llevados al Mercado Central de Huánuco); en Sandia (Puno), en donde en el 2006 se trató de vender un osezno de seis meses cazado en Quiaca a US\$ 50, pero como su venta resultó difícil, fue criado como mascota; en Mariano Melgar (Arequipa), donde se vendieron dos oseznos provenientes de Madre de Dios a US\$ 1.000 cada uno, y en la feria de Motupe (Lambayeque), en el 2002, se vendió un oso de tres años a US\$ 87.

\section{Discusión}

\section{Venta de partes}

En los países asiáticos existe un mercado organizado de partes de osos. En Indonesia y Singapur se ha reportado la venta de partes del oso malayo Helarctos malayanus como la vesícula biliar, las garras, la piel y los dientes a manera de souvenir para los turistas. En Singapur, las garras del oso malayo se venden entre US\$1,4 y 6,6 , las patas, a US\$11,1 y los caninos entre US\$2,2 y 11 (WSPA, 2002b). Además, la carne y las patas de oso se consumen en restaurantes de China, Hong Kong y Japón, entre otros (Mills \& Servheen, 1994). En Indonesia, una porción de sopa hecha con patas de oso malayo o del oso negro asiático Ursus thibetanus para 10 personas tiene un costo de US $\$ 220,2 \mathrm{y}$, en algunos casos, se debe hacer el pedido con 15 días de anticipación (WSPA, 2002b).

Con respecto al oso andino, se ha señalado como una amenaza adicional para su conservación el tráfico ilegal de partes, en particular de garras, dientes y vesículas biliares, de gran demanda en los países asiáticos (Peyton, et al., 1998). En Colombia no se ha podido determinar el alcance de este mercado, pero no se le considera un problema serio (Orejuela \& Jorgenson, 1996). En Mérida (Venezuela) algunas partes de osos que se emplean como productos curativos o como amuletos se venden directamente $\mathrm{y}$, en otros casos, deben pedirse con anticipación (Herrera, et al., 1994), y en Bolivia, en el Mercado de las Brujas de La $\mathrm{Paz}$, se ha registrado la venta de talismanes con pequeñas porciones de huesos o lengua, así como grasa sólida para curar el reumatismo a US\$ 2,3 (Paisley, 2001).

A pesar de que el tráfico de fauna silvestre es el resultado de un fenómeno de escala mundial que se lleva a cabo en países en desarrollo en respuesta a las dinámicas de la demanda en los países desarrollados (Moyle, 1998), el tráfico de partes de oso tiene una demanda básicamente local, a excepción de los escasos registros de venta de vesícula biliar a personas de origen asiático en Ecuador y en el Perú, caso que se discute más adelante.

En los mercados visitados, el costo de las partes variaba considerablemente dependiendo del tipo de comprador (local o foráneo, en especial de la capital). Por ejemplo, la pata del oso en Piura tenía un precio entre US\$ 7,14 y 28,5 (Amanzo, et al., 2007a), en Lambayeque, entre US\$ 5,8 a 17,4, en La Libertad, de US\$ 4,4, en San Martín, entre US\$ 8,7 a 29, en Cajamarca, de US\$ 8,7 y en Cusco, de US\$ 29.

La venta de partes se encuentra estrechamente relacionada con su uso curativo. En el Perú, este uso parece remontarse a la época inca, con los Qallahuayas (curanderos, chamanes, brujos, magos y médicos), quienes continuaron curando con los mismos métodos en la época colonial. Esta medicina se basaba principalmente en un proceso de sugestión que se valía de las "fuerzas espirituales" para dominar la enfermedad o el espíritu maligno que la engendraba, lo que explica el uso de amuletos y talismanes con partes de osos dotados de "fuerza curativa" (Cavero, 1988). En otros casos, se empleaba la grasa del oso para curar los tumores (Baumann, 1966) y hasta fines del siglo XVIII las partes del oso seguían usándose como medicinas (Lequanda, 1793a, 1793b). A pesar de los siglos que han transcurrido, estas influencias culturales continuaron actuando en el siglo XX (Valdizán \& Maldonado, 1922), y persisten entre los pobladores rurales y urbanos actuales, quienes compran las partes del oso andino con el fin de curar sus enfermedades, alejar a los "malos espíritus", o como afrodisíaco o sustancia fortificante (Figueroa, 2003, 2008), uso que se incrementa debido a la insuficiencia y la ineficacia de la atención médica formal.

A la grasa se le atribuye la propiedad de curar enfermedades en los pulmones, los riñones, el hígado y los huesos, los dolores musculares, el "susto" y el herpes, así como la de cicatrizar heridas, entre otras. También la aplican para que los niños crezcan "fuertes y sanos" y los hombres tengan "más fuerza". A la sangre también se le da este uso, por lo que se toma fresca. Los huesos son utilizados en el tratamiento de la artritis, como fuente de calcio y para mantener en buen 
estado el útero; el hueso peneano se usa específicamente como afrodisíaco, al igual que los testículos, en tanto que la garra se usa para curar la epilepsia, la piel para problemas de reumatismo y dislocaduras, y la hiel para curar el paludismo y la epilepsia, mientras que el concentrado de la cabeza hervida del oso se toma para aliviar los calambres y los espasmos musculares (Figueroa \& Stucchi, 2013).

El hecho de que el mayor volumen de venta de partes se de en los mercados de las regiones del norte (Lambayeque, Piura, San Martín y Cajamarca) coincide con la circunstancia de ser estas las áreas donde las personas dicen conocer una gran cantidad de sus propiedades curativas. Además, en el norte existe una gran influencia de los curanderos, quienes usan partes de animales en sus tratamientos (Figueroa, 2003). Para el caso particular de Lambayeque, el mayor volumen de ventas de partes también se relacionaría con la larga historia de caza del oso (Baca, 1957), y con sus usos alimenticios y medicinales, difundidos por los indígenas y asimilados por los españoles y sus descendientes (Lequanda, 1793b). Asimismo, en la región confluyen las principales vías terrestres de acceso a Amazonas, San Martín y Cajamarca, donde también existe una importante población de esta especie, y de donde proceden las partes de oso para la venta en los mercados de Chiclayo (Lambayeque), los cuales se reabastecen cada cierto tiempo debido a la demanda. Chiclayo también ha sido identificado como centro de acopio del comercio de otras especies de fauna silvestre en el norte del Perú (Ríos, et al., 2008).

A pesar de las leyes peruanas e internacionales que protegen al oso andino, el comercio de sus partes continúa realizándose en los principales mercados del país de forma continua y extensiva. En un estudio reciente en Cajamarca y Amazonas, se pudo encontrar que el mercado de las partes del oso y de otras especies en peligro, como el cóndor andino, continúa abasteciéndose (Figueroa, et al., 2013). Por otro lado, la venta de partes del cóndor andino también se viene realizando en algunos mercados de Cusco (Williams, et al., 2011).

\section{Venta de vesícula biliar del oso andino}

La vesícula del oso y sus derivados, tales como el "polvo seco de bilis", los "cristales de bilis", las pastillas y los tónicos, tienen una gran demanda en diversos países del mundo como Rusia (Chestin, 1998), Canadá, EE.UU., Indonesia, Japón, Malasia, Singapur, Taiwán y Australia (WSPA, 2002b) (Figura 2). Estos productos se consumen para prevenir enfermedades y, además, se les atribuyen propiedades curativas para la conjuntivitis, el asma, los cálculos, la hepatitis, la sinusitis, las hemorroides, la sífilis, el cáncer (de pulmón, hígado, intestino, estómago y útero), las fiebres altas, las convulsiones y el parasitismo (Servheen, 1990; Highley \& Chang, 1994; Mills, et al., 1995; Baik, 2001; Ge Gabriel, 2001; Lee, 2001; Govind \& Ho, 2001;
Dainobu, et al., 2002). Según algunas investigaciones, las propiedades curativas de la bilis se basan en la acción del ácido tauroursodesoxicólico (AUDC), que se encuentra en casi todas las especies de úrsidos a excepción del panda gigante, aunque con niveles más altos en los osos negros, los pardos y los polares (Baik, 2001). Con el objetivo de obtener una mayor cantidad de bilis para cubrir la demanda internacional, en 1984 Corea del Norte creó granjas de osos con el fin de extraerles continuamente la bilis, principalmente de osos negros asiáticos (Raloff, 2005). Este mecanismo llegó rápidamente a China, país que se convirtió en el principal extractor de bilis mediante este método en el mundo (Fan \& Song, 2001).

Sin embargo, aunque los osos presentan el AUDC en niveles significativos, este puede sintetizarse a partir del ácido cólico, abundante en otras especies como cerdos y vacas (de donde lo extraen las marcas Actigall, Deursil, Ursosan, Ursofalk, Urso y UrsoForte) (Foley, et al., 2011), obteniendo los mismos resultados con menores costos y, sobre todo, sin disminuir las poblaciones silvestres de osos (Baik, 2001). Otra alternativa es el uso de hierbas medicinales como Lobelia chinensis (Campanulaceae), Hedyotis diffusa (Rubiaceae), Patrinia scabiosifolia (Caprifoliaceae), Taraxacum mongolicum (Asteraceae) y Viola yedoensis (Violaceae), entre otras (Pong, et al., 2001; WSPA, 2005).

Se sabe que el comercio local de partes de oso tiene una larga historia en los Andes, pero la evidencia de la demanda de los mercados asiáticos es reciente. En Ecuador se tienen reportes de compradores coreanos de vesícula del oso andino en Cambaye-Coca y Cotacachi-Cayapas, quienes ofrecieron US\$ 115 por cada una en la ocasión reseñada (Peyton, et al., 1998; WSPA, 2002a). En el Perú, su venta en los mercados se ha dado muy raramente, en este estudio solo se encontró una vesícula deshidratada en Chiclayo, lo que se relaciona con el escaso uso que le dan los pobladores a esta parte del oso. Solo tres personas señalaron haber usado la bilis para curar la epilepsia, los ataques cardiacos y el paludismo en San Martín, La Libertad y Lambayeque, respectivamente (Figueroa, 2008). Sin embargo, como ya se mencionó, sí se obtuvieron dos reportes de su venta a compradores asiáticos en Amazonas y Cusco. No sería la primera vez que se relaciona la compra de partes de fauna silvestre en el Perú con compradores provenientes de Asia. Por ejemplo, se sabe que en el 2004 compradores coreanos contrataron a pescadores de Ica para la cacería del lobo marino chusco Otaria flavescens en la isla San Gallán, con el fin de extraerle el hueso peneano para su uso como afrodisíaco (personal de la Reserva Nacional de Paracas, comunicación personal, 2005).

Con base en la información obtenida por los vendedores, los precios de la vesícula en el Perú serían muy variables: desde US\$ 5,8 en Lambayeque hasta US\$ 580 en Cusco (Tabla 6). 


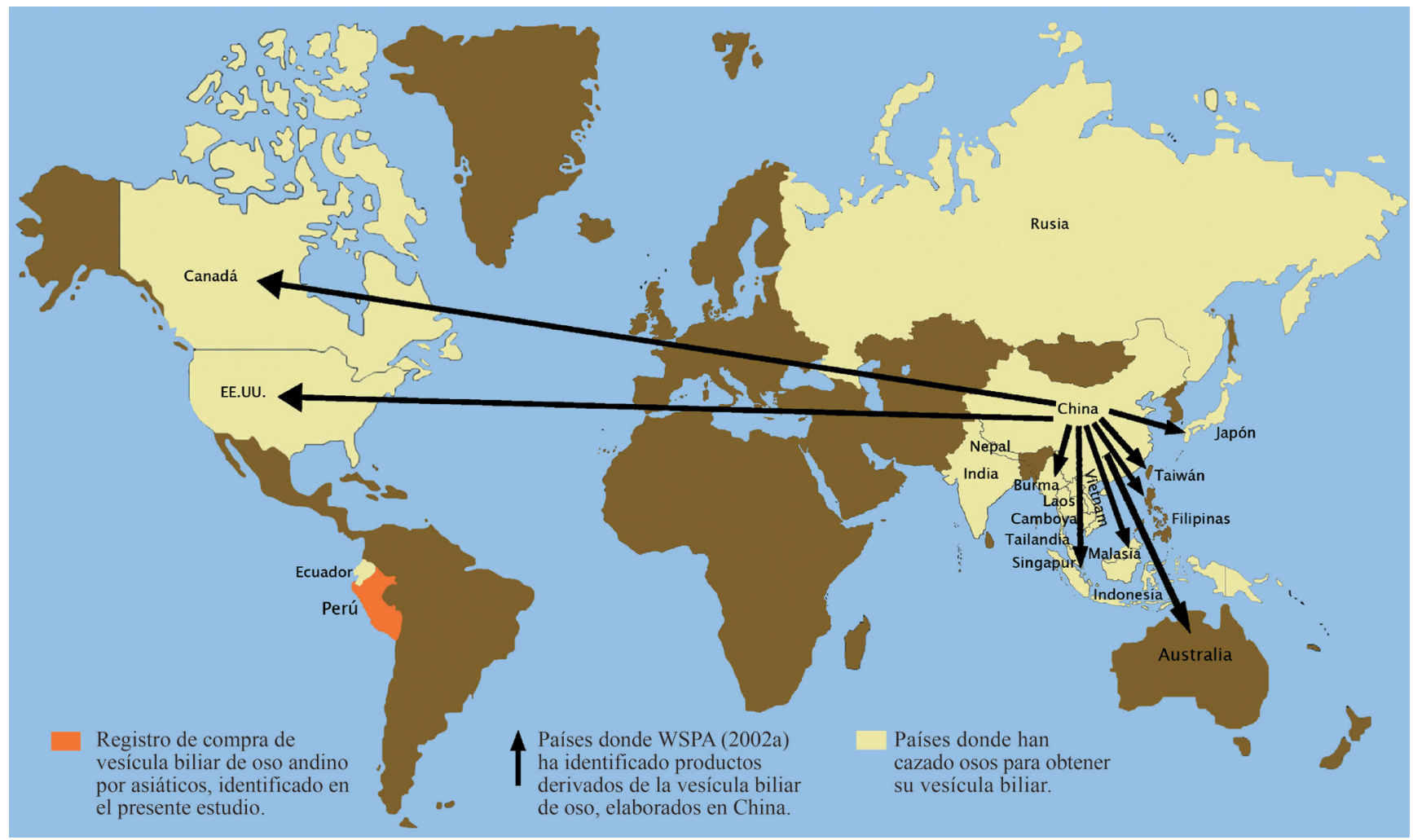

Figura 2. Comercio de la vesícula biliar de osos (Fuente: editado de WSPA, 2002a).

Tabla 6. Comparación de los costos de la vesícula biliar de osos en diversos países

\begin{tabular}{lccccc}
\hline País & Mayor precio (US\$) & Menor precio (US\$) & País & Mayor precio (US\$) & Menor precio (US\$) \\
\hline Perúł & 580 & 5,8 & Taiwan* & 1.000 & 60 \\
Ecuador§ & 115 & & Malasia* & 5.263 & 8,15 \\
Canadá y EE.UU.* & 650 & 50 & Singapur* & 290 & 8,25 \\
Australia* & 10,9 & & China* & 50 & 30 \\
Japón* & 168 & 25 & Indonesia* & 111 & 8,3 \\
\hline
\end{tabular}

Fuentes: * =WSPA, 2002b; $\S=$ Peyton, et al., 1998; $\$=$ Presente estudio

En un estudio se encontró el mismo fenómeno en los países del este y sudeste asiático, donde los precios por gramo de vesícula biliar de oso fluctuaban entre US\$ 3 y 210 en 1990 y 1991 (Mills \& Servheen, 1994). Una posible explicación sería que en algunas ocasiones se venden vesículas de cerdo o vaca como si fueran de oso, ya que son similares (Highley \& Chang, 1994; Chestin, 1998); además, en el caso del Perú, el costo en Lambayeque se debería a la poca demanda local.

\section{Productos elaborados bajo el nombre de frotación de oso}

A diferencia de los países asiáticos donde se elaboran diversos productos a base de vesícula biliar, en el Perú se encontró una pomada conocida como frotación de oso, que es un producto común en la medicina tradicional. De las 16 diferentes presentaciones observadas, el $81,2 \%$ no llevaba ningún registro de inscripción o era falso según la Superintendencia Nacional de Administración Tributaria (SUNAT, 2008), mientras que en el $18,8 \%$ sí se verificó la existencia del registro. Uno de ellos remitía a una empresa de Lima, y dos, a personas naturales de Lima y Junín. Según el código de la Clasificación Industrial Internacional Uniforme (CIIU), este tipo de registro corresponde a "venta al por mayor de otros productos" (CIIU 51906) y a "otros tipos de venta al por menor" (CIIU 52391), por lo que es probable que la SUNAT de Perú no tuviera conocimiento sobre la venta de la frotación de oso por parte de estas personas naturales 
y de la empresa. Es presumible que estos productos sean elaborados en la capital y luego se distribuyan a otras áreas del país. El mercado La Parada de Lima es uno de los principales centros de acopio de todo tipo de productos en el Perú; según los vendedores de este establecimiento, los dos mercadillos "Hierba Santa 1 y 2", que albergan aproximadamente 100 puestos de venta de productos de medicina tradicional, abastecen a su vez a las tiendas más pequeñas, principalmente en la capital, y a los curanderos más famosos del país.

Debido a que la frotación de oso se elabora en Lima, su costo en la capital fue el más bajo y fluctúo entre US\$ 0,15 y 0,3 $(15 \mathrm{~g})$ y US\$ 0,7 (28 g), mientras que este mismo producto en otras regiones tuvo un costo más elevado. Los precios de la presentación de $15 \mathrm{~g}$ fueron de US\$ 0,9 a 1,16 en Ancash, de US\$ 0,6 en Arequipa y Cajamarca, de US\$ 0,7 en Piura y Huánuco y de US\$ 0,7 a 0,9 en Cusco, en tanto que la de 28 $\mathrm{g}$ fue de US\$ 1,5 en Piura y Huánuco.

En los productos así llamados de frotación de oso, el componente principal es la grasa de oso, la cual se mezcla con alcanfor, mentol, salicilato de metilo y excipientes. Su bajo costo (15 g a US $\$ 0,15$ y 0,9$)$, y sus características físicas (color, olor, textura) iguales a otras pomadas que dicen contener grasa de mula, culebra o iguana, indicarían que se trata de falsificaciones. De todos estos productos, incluida la frotación de oso, se asegura que curan problemas de artritis, reumatismo, dislocaduras, fracturas, "recalco", relajamiento muscular, lumbago, bronquitis, tos, resfrío, várices, hematoma, calambre, "choques de aire", inflamación de los ovarios y amígdalas, dolores de cabeza, de espalda, de pecho, de huesos y musculares. Todo esto indica que la frotación de oso usa el nombre de la especie como un "gancho", debido a la gran fama de la grasa del oso como sustancia medicinal. Además de su bajo precio, la gran demanda de la frotación de oso se debe a la extensa lista de enfermedades que cura, entre las que se destacan las infecciones de las vías respiratorias, las cuales son una de las principales causas de mortalidad en el Perú (19\% de los casos) (MINSA, 2013). Es posible que la frotación de oso ocasione algún tipo de disminución del dolor, ya que entre sus componentes principales está el salicilato de metilo, un éster empleado como agente aromatizante que se absorbe a través de la piel, se hidroliza y se transforma en ácido salicílico, el cual actúa como analgésico. Además, contiene extracto de árnica, que tiene propiedades de antiséptico, astringente, antiinflamatorio, anticoagulante y estimulador de la circulación y posiblemente también contenga extractos de otras plantas con propiedades antiinflamatorias. Sin embargo, su uso podría ser contraproducente para la persona que presenta alguna enfermedad, porque, lejos de curarla, funcionaría solo como un paliativo.

\section{Venta de oseznos}

Existen reportes del comercio de oseznos de oso malayo y oso negro asiático en países como Laos, Camboya, Myanmar y Vietnam (Kemf, et al., 1999). Otras personas adquieren osos con el fin de usarlos en exhibiciones públicas como los "osos danzantes" en Bulgaria, las peleas de osos y perros en Pakistán, y también como atractivo para que los turistas se tomen fotografías en Rusia, o para que ejecutan piruetas en los circos (Kemf, et al., 1999).

Desde inicios del siglo XX distintas especies de osos han sido utilizadas como atracción pública en el Perú, entre ellos el oso andino (Figueroa \& Stucchi, 2013). Comúnmente las crías son atrapadas después de la cacería de la madre. En algunos casos, los mismos cazadores se las quedan como mascotas o las venden a circos, a coleccionistas particulares y a zoológicos (Figueroa \& Stucchi, 2005; La República, 2012; Figueroa, et al., 2013; Inkaterra Asociación, 2013). Este hecho pone en evidencia la violación de la Ley $\mathrm{N}^{\circ}$ 29763, Forestal y de Fauna Silvestre, en cuyas disposiciones complementarias finales se prohíbe la exhibición y empleo de especímenes de fauna silvestre, nativas y exóticas en espectáculos circenses itinerantes (Congreso de la República, 2011).

Cuando los oseznos son criados como mascotas, entre el tercer y cuarto año son sacrificados para el consumo (principalmente en los pueblos más alejados de las ciudades) o vendidos, pues su mantenimiento se encarece y también porque quienes los tienen temen que les hagan daño (Figueroa \& Stucchi, 2005). Los casos que aquí se reseñan fueron reportados en Cutervo (Cajamarca), Pozuzo (Pasco), Lucmabamba (Cusco) y Chachapoyas (Amazonas); en los dos primeros casos, los animales fueron sacrificados, el tercero fue decomisado y el último se vendió en el mercado de Chiclayo (Figueroa, et al., 2013). Lo mismo ocurre con el oso malayo en Singapur: una vez adquirido, el dueño lo mantiene como mascota hasta que se convierte en una molestia debido a su tamaño y a sus crecientes necesidades de alimentación. Por este motivo los matan y extraen la vesícula biliar para su uso como medicina (WSPA, 2002b). En Ecuador se han realizado decomisos de osos andinos cuyo destino final suelen ser los mercados locales donde, en muchos de los casos, se comercializa su carne (EI Telégrafo, 2012).

Los precios de los oseznos en los mercados visitados variaban mucho: en Arequipa costaban US\$1.000, en Mochumí (Lambayeque), US\$ 500, en Motupe (Lambayeque), US\$ 87, en Mal Paso (Pasco), US\$ 29 y en Sandia (Puno), US\$ 50. La diferencia entre los precios de Arequipa y Mochumí y los de los demás lugares puede deberse a que en los dos primeros sitios los oseznos iban a ser vendidos en grandes ciudades (Arequipa y Chiclayo), donde existe un mayor 
movimiento económico y, en cambio la venta en Motupe, Mal Paso y Sandia se realizó cerca al área de extracción. Un caso similar ocurre con los osos malayos, los cuales se venden como mascotas entre los 3 y los 24 meses de edad a precios que varían dependiendo de quien realice la venta. $\mathrm{Si}$ son vendidos directamente por el cazador, el precio puede ser entre US\$ 11 y 67 por animal, y si se venden en una tienda, su valor asciende hasta US\$ 222 (WSPA, 2002b).

\section{Problemática del tráfico de fauna silvestre en el Perú}

Actualmente, en el Perú solo se conoce el valor aproximado de la exportación legal de fauna silvestre (categoría 0106, según las Estadísticas de Comercio para el Desarrollo Internacional de las Empresas, animales vivos), en el transcurso de los años, pasando de US\$ 436.000 en el 2011 a US\$ 733.000 en el 2012. La demanda principal se ha concentrado en China (69,4 \%), España $(9,4 \%)$, EE.UU. $(7,5 \%)$ y Tailandia $(2,9 \%)$. Los grupos que más se exportaron fueron los reptiles $(62,1 \%)$, las aves $(5,7 \%)$ y los primates $(2,1 \%)$ (International Trade Center, 2014). Sin embargo, se desconoce el alcance del comercio de partes y ejemplares de la fauna silvestre tanto en el mercado nacional como en el internacional, pero si nos basamos en los datos de Colombia, un país de gran biodiversidad donde se presume que el volumen del tráfico ilegal es de gran magnitud y solo se decomisa entre 1 y $10 \%$ del total (Baptiste, et al., 2002), en el Perú dicho tráfico tendría un alcance similar. De igual manera, el impacto de este comercio sobre la fauna silvestre sería muy significativo si se toma en cuenta que por cada animal vivo que llega a una plaza de mercado en EE.UU., 10 han sido capturados en estado silvestre (Aguirre, 2000). En el Perú, el Instituto Nacional de Recursos Naturales (Ríos, et al., 2008) calculó que nueve de cada 10 aves (loros, pericos, paseriformes) capturadas y trasladadas de manera inadecuada mueren antes de llegar a su destino, mientras que cuatro de cada cinco primates pequeños mueren bajo circunstancias similares. Asimismo, los cazadores extraen a pedido de los vendedores un número adicional de animales, a manera de consignación, para compensar los animales muertos o decomisados (Ríos, et al., 2008).

Como se ha señalado en varias oportunidades, este comercio se realiza ante la vista y la indiferencia de las autoridades y la población civil, lo que podría deberse a la suma de varios factores: la mala administración de los recursos económicos, lo que conlleva a una escasa supervisión; la falta de personal capacitado tanto en la Policía Ecológica como en las áreas ambientales de los Gobiernos Regionales (debido, en estas últimas, a la descentralización que les confiere la facultad de tomar decisiones sobre qué hacer con los animales incautados); el limitado control policial en los aeropuertos y terminales terrestres; la falta de programas de sensibilización y educación ambiental en torno a estos temas; la impunidad de los traficantes en los procesos judiciales debido a la ignorancia de los jueces sobre el tema, a pesar de que desde el 2008 se crearon las Fiscalías Especializadas en Materia Ambiental (Resolución de la Junta de Fiscales Supremos No 038-2008-MP-FNJFS); los actos de corrupción en que se ha visto involucrado personal del Estado, tal como sucede en el tráfico ilegal de madera; el hecho de que los traficantes siempre están ideando nuevas modalidades; la falta de centros de rescate y rehabilitación, y el conocimiento insuficiente para caracterizar, analizar y evaluar la estructura y la dinámica del tráfico ilegal de especies silvestres y así tomar medidas de protección efectivas.

En el caso del oso andino, su comercio pone en evidencia la violación de las regulaciones de la CITES, así como del artículo 308 del Título XIII del Código Penal, y del Decreto Legislativo $\mathrm{N}^{\circ} 635$ de 1991 relativo a los delitos contra los recursos naturales y el medio ambiente, donde se señala una pena privativa de libertad no menor de uno ni mayor de tres años para los infractores. Sin embargo, a diferencia de otros países donde los traficantes son arrestados y enjuiciados por el comercio de individuos y partes de oso (Williamson, 2002; Kishor Gupta, et al., 2007), en el Perú no ha sucedido así.

\section{Conclusiones}

La compra de las partes del oso andino se hace básicamente a nivel local, tanto por parte de pobladores rurales como urbanos, con fines curativos, mágicos, afrodisíacos y alimenticios. Se obtuvieron, además, dos registros de compra de vesícula biliar por parte de personas de origen asiático en Cusco y Amazonas.

El mayor volumen de venta de partes se registró en los mercados de las regiones del norte, Lambayeque, Piura, San Martín y Cajamarca, en sitios que coinciden con las áreas en donde las personas dicen conocer una gran cantidad de sus propiedades medicinales y afrodisiacas. En el caso particular de Lambayeque, allí confluyen las principales vías terrestres de acceso desde Amazonas, San Martín y Cajamarca, además de ser una zona con una población significativa de esta especie desde la cual se envían las partes del oso para su venta en los mercados de Chiclayo.

Existen al menos diez leyes peruanas expedidas desde el año 1970 que protegen al oso andino, además de las normas internacionales de la IUCN y la CITES. Sin embargo, el comercio de sus partes se realiza en los principales mercados del país ante la vista de las autoridades, quienes poco han podido hacer para contrarrestar este problema. 


\section{Información suplementaria}

Figura 1S. Empaques de la "frotación de oso" a la venta en diversos mercados del Perú (Foto: Judith Figueroa)

Figura 2S. Grasa y aceite de oso a la venta en diversos mercados del Perú (Foto : Judith Figueroa)

Figura 3S. Pata posterior de un oso andino a la venta en Cajamarca (Foto: Judith Figueroa)

Figura 4S. Hueso peneano y testículos de un oso andino a la venta en Nueva Cajamarca, San Martín (Foto: Judith Figueroa)

Figura 5S. Vesícula biliar deshidratada de un oso andino a la venta en Chiclayo, Lambayeque (Foto: Judith Figueroa)
Figura 6S. Partes de un gallito de las rocas, de un cóndor andino y un pico de tucán Ramphastos sp. a la venta en Chiclayo, Lambayeque (Foto: Judith Figueroa)

Figura 7S. Patas de tapir de páramo a la venta en Chiclayo, Lambayeque (Foto: Judith Figueroa)

Figura 8S. Pieles de mamíferos a la venta en Pucallpa, Ucayali (Foto: Judith Figueroa)

Figura 9S. Osezna decomisada en Mochumí, Lambayeque (Foto: Judith Figueroa)

\section{Agradecimientos}

Un agradecimiento especial a Marcelo Stucchi, por su apoyo en las entrevistas en los mercados. A Siegfried Kastl, de la Cooperación Técnica Alemana, por el financiamiento del presente estudio. A Trinidad Tapia y Alejandro Zegarra, por la información brindada. A Víctor Watkins, de la World Society for the Protection of Animals, y a Lorena Hidalgo, de Traffic América del Sur, por el envío de bibliografía. A Roxana Rojas-Vera Pinto, por la elaboración de la Figura 1.

\section{Conflicto de interés}

La autora declara que no tiene conflicto de interés.

\section{Bibliografía}

Aguirre, A. 2000. El comercio ilegal de fauna silvestre y técnicas forenses aplicadas. p. 249-254. En: F. Nassar-Montoya \& R. Crane (eds.). Actitudes hacia la fauna en Latinoamérica. Humane Society Press, Washington, USA.

Amanzo, J., Chung, C., Zagal, M. \& Pacheco, V.2007a. Evaluación del oso andino Tremarctos ornatus en Piura y Cajamarca. Serie de Publicaciones de Flora y Fauna Silvestre, Instituto Nacional de Recursos Naturales. Lima, Perú.

Amanzo, J., Mendoza, W., Chung, C. \& Villalobos, M. 2007b. Evaluación de oso andino Tremarctos ornatus en Amazonas. Serie de Publicaciones de Flora y Fauna Silvestre, Instituto Nacional de Recursos Naturales. Lima, Perú.

Baca, V. (1957, enero, 15). Recuerdos de un Cazador. Diario "El País". Chiclayo, Perú.

Baik, K. 2001. New developments in the use of synthesised bear bile in medicine. En D. Williamson \& M. J. Phipps (eds.). Proceedings of the Third International Symposium on the Trade in Bear Parts. Traffic East Asia. Seoul, Republic of Korea.

Baptiste, L. G., Polanco, R., Hernández, S. \& Quiceno, M. P. 2002. Fauna silvestre de Colombia: historia económica y social de un proceso de marginalización. p. 295-340. En: A. Ulloa (ed.). Rostros culturales de la fauna. Las relaciones entre los humanos y los animales en el contexto colombiano. Instituto Colombiano de Antropología e Historia-ICANH. Bogotá DC.

Baumann, H. 1966. Oro y dioses del Perú. Editorial Juventud. Barcelona, España.

Cavero, G. 1988. Supersticiones y Medicina Quechuas. Editorial Libertad EIRL. Trujillo, Perú.

Chestin, I. E. 1998. Trade in Brown Bear Gall Bladders in Russia. Ursus, 10: 161-166.

Congreso de la República. 2011. Ley Forestal y de Fauna Silvestre. Ley No 29763. Diario Oficial El Peruano, Normas Legales: 446980 - 447004.

Convención sobre el Comercio Internacional de Especies Amenazadas de Fauna y Flora Silvestres - CITES. 2013. Apéndices I, II y III. Disponible en http://www.cites.org/ esp/app/2013/S-Appendices-2013-06-12.pdf

Dainobu, N., Togawa, K., Sakamoto, M. 2002. Japan's illegal trade in bear products. Japan Wildlife Conservation Society. Japón.

El Telégrafo. (2012, octubre, 21). Morona y Zamora emprenden lucha contra tráfico de especies. Disponible en: http:// www.telegrafo.com.ec/regionales/regional-sur/item/ morona-y-zamora-emprenden-lucha-contra-trafico-deespecies.html

Fan, Z. \& Song, Y. 2001. The development of bear farming in China. En D. F. Williamson \& M. J. Phipps (eds.). Proceedings of the Third International Symposium on the Trade in Bear Parts. (pp. 100-109). Traffic East Asia. Seoul, Republic of Korea.

Figueroa, J. 2003. Cacería del oso andino en el Perú: etnozoología y comercio. Cooperación Técnica Alemana - GTZ/ FANPE, Proyecto Oso Andino Perú. Lima, Perú.

Figueroa, J. 2008. Cacería del oso andino en el Perú. Memoria para la obtención del Diploma de Estudios Avanzados. Centro 
Iberoamericano de la Biodiversidad - CIBIO, Universidad de Alicante. Alicante, España.

Figueroa, J. \& Stucchi, M. 2002. Situación actual del oso andino en el Santuario Histórico de Machu Picchu y zonas adyacentes. Cusco, Perú. Cooperación Técnica Alemana GTZ-FANPE, Proyecto Oso Andino Perú. Lima, Perú.

Figueroa, J. \& Stucchi, M. 2005. Registro del oso andino en cautiverio en el Perú y algunos alcances para su mantenimiento. Reporte de Investigación $\mathrm{N}^{\mathrm{o}} 2$. Asociación Ucumari. Lima, Perú.

Figueroa, J. \& Stucchi, M. 2013. Visión histórica de la cacería del oso andino en América del Sur. En J. Figueroa (ed.). El hermano oso andino. Su presencia en la cultura de América. (pp. 57-84). Asociación para la Investigación y Conservación de la Biodiversidad (AICB). Lima, Perú.

Figueroa, J., Stucchi, M. \& Rojas-VeraPinto, R. 2013. Redes de conectividad: el oso andino como una especie importante en la conservación del bosque seco del Marañón (Cajamarca y Amazonas, Perú). Cooperación Internacional Alemana GIZ, Asociación para la Investigación y Conservación de la Biodiversidad - AICB. Lima, Perú.

Foley, K. E., Stengel, C. J. \& Shepherd, C.R. 2011. Pills, powders, vials and flakes: The bear bile trade in Asia. TRAFFIC Southeast Asia, Petaling Jaya, Selangor, Malaysia.

Ge Gabriel, G. 1999. A bitter medicine: The use of bear bile in China. En D. F. Williamson \& M. J. Phipps (eds.). Proceedings of the Third International Symposium on the Trade in Bear Parts. (pp. 116-120). Traffic East Asia. Seoul, Republic of Korea.

Govind, V. \& Ho, S. 2001. Report on the trade in bear gall bladder and bile products in Singapore. Animal Concerns Research and Education Society - ACRES, World Society for the Protection of Animals - WSPA.

Herrera, A. M., Nassar, J., Michelangeli, F., Rodríguez, J. \& Torres, D. 1994. The spectacled bear in the Sierra Nevada National Park of Venezuela. International Conference on Bear Research and Management, 9(1): 149-156.

Highley, K. \& Chang, S. 1994. Bear farming and trade in China and Taiwan. Earthtrust Taiwan for the Humane Society of the United States and Humane Society International.

Inkaterra Asociación. 2013. Arribo de dos hembras de oso de anteojos a Machu Picchu. Disponible en: https://www. facebook.com/media/set/?set=a.571877236195904.107374 $1825.139271279456504 \&$ type $=3$.

International Fund for Animal Welfare - IFAW. 2013. Seven tons of seized ivory add up to bloody few weeks for elephants. Disponible en: http://www.ifaw.org/unitedstates/news/seven-tons-seized-ivory-adds-bloody-fewweeks-elephants.

International Union for Conservation of Nature - IUCN. 2013. IUCN Red List of Threatened Species. Version 2013.1. Disponible en: http://www.iucnredlist.org/details/22066/0
International Trade Center. 2014. Trade Map. Estadísticas de comercio para el desarrollo internacional de las empresas. Disponible en: http://www.trademap.org/Country SelProduct Country.aspx

Kemf, E., Wilson, A., Servheen, C. 1999. Bears in the wild. WWF Species Status Report.

Kishor Gupta, B., Singh, R., Satyanarayan, K. \& Seshamani, G. 2007. Trade in bears and their parts in India: Threats to conservation of bears. En D. F. Williamson (ed.). Proceedings of the Fouth International Symposium on the Trade in Bear Parts. (pp. 50-60). Traffic East Asia. Tokio, Japón.

La República. (2012, noviembre, 15). Lambayeque: oso de anteojos fue rescatado de cautiverio. Disponible en: http:// www.larepublica.pe/15-11-2012/lambayeque-oso-deanteojos-fue-rescatado-de-cautiverio\#! foto1.

Lee, Y. 2001. The use of bear bile as medicine versus tonic. En D. F. Williamson \& M. J. Phipps (eds.). Proceedings of the Third International Symposium on the Trade in Bear Parts. (pp. 122-126). Traffic East Asia. Seoul, Republic of Korea.

Lequanda, de J. I. 1793a. Continuación de la descripción geográfica de la ciudad y partido de Truxillo. Tomo VIII, $\mathrm{N}^{\circ}$ 249, folios 52-59. Mercurio Peruano, 23 de mayo de 1793. Lima, Perú.

Lequanda, de J. I. 1793b. Continuación de la descripción geográfica del Partido de Piura perteneciente á la Intendencia de Truxillo. Tomo VIII, No 264, folios 175-182. Mercurio Peruano, 14 de julio de 1793. Lima, Perú.

Mancera Rodríguez, N. J. \& Reyes García, O. 2008. Comercio de fauna silvestre en Colombia. Revista Facultad Nacional de Agronomía Medellín, 61(2): 4618-4645.

Mills, J. A. \& Servheen, C. 1994. The Asian trade in bears and bear parts: Impacts and conservation recommendations. International Conference on Bear Research and Management, 9(1): 161-167.

Mills, J. A, Chan, S., Ishihara, A. 1995. Species in danger. The bear facts: The East Asian market for bear gall bladder World Wide Fund for Nature, Washington, D.C., USA.

Ministerio de Agricultura - MINAG. 2004. Aprueban categorización de especies amenazadas de fauna silvestre y prohíben su caza, captura, tenencia, transporte o exportación con fines comerciales. Decreto Supremo No 034 2004-AG. Diario Oficial El Peruano, Normas Legales: 276853-276856.

Ministerio de Agricultura - MINAG. 2013. Nueva ley forestal y de fauna silvestre. Por un aprovechamiento sostenible, equitativo y competitivo de los bosques en el Perú. Lima, Perú.

Ministerio de Salud - MINSA. 2013. Principales causas de mortalidad por sexo, Perú - año 2011. Disponible en: http:// www.minsa.gob.pe/estadisticas/estadisticas/Mortalidad/ Macros.asp?00.

Moyle, B. 1998. The bioeconomics of illegal wildlife harvesting: An outline of the issues. Journal of International Wildlife Law and Policy, 1(1): 95-111. 
Orejuela, J. \& Jorgenson, J. 1996. Plan de acción del oso andino. Encuentro nacional sobre conservación y manejo del oso andino. Ministerio del Medio Ambiente. Bogotá, Colombia.

Paisley, S. 2001. Andean bears and people in Apolobamba, Bolivia: Culture, conflict and conservation. A thesis submitted for the degree of Doctor of Philosophy. Durrell Institute of Conservation and Ecology. University of Kent, Canterbury. UK.

Peyton, B., Yerena, E., Rumiz, D., Jorgenson, J. \& Orejuela, J. 1998. Status of wild Andean bears and policies for their management. Ursus, 10: 87-100.

Pong, S, Lo, Y. W., Ho, K. C. 2001. Herbal alternatives to bear bile. En D. F. Williamson \& M. J. Phipps (eds.). Proceedings of the Third International Symposium on the Trade in Bear Parts. (pp. 154-1579. Traffic East Asia. Seoul, Republic of Korea.

Pulido, V. 1991. El libro rojo de la fauna silvestre del Perú. Instituto Nacional de Investigación Agraria y Agroindustrial. Lima, Perú.

Raloff, J. 2005. A galling business: The inhumane exploitation of bears for traditional Asian medicine. Science News, 168(16): 250-252.

Ríos, L., Riva, F., Canaquire, L. 2008. Reporte situacional del tráfico ilegal de fauna silvestre en la región nororiental del Perú. ProNaturaleza. Lima, Perú.

Salazar, J. \& Anderson, S. 1990. Informe sobre el estado actual del conocimiento del oso andino en Bolivia. Ecología en Bolivia, 15: 3-23.
Servheen, C. 1990. The status and conservation of the bears of the world. VIII International Conference of Bear Research and Management. Victoria, British Columbia, Canada.

Superintendencia Nacional de Administración Tributaria SUNAT. 2008. Consulta RUC. Disponible en: http://www. sunat.gob.pe/cl-ti-itmrconsruc/jcrS00Alias.

Valdizán, H. \& Maldonado, A. 1922. La medicina popular peruana. Tomo I. Consejo Indio de Sudamérica- CISA. Imprenta Torres Aguirre. Lima, Perú.

Villena Arboccó, M. 2013. Tráfico ilegal de fauna silvestre en el Perú. Revista Virtual FaunaVet-Perú, 3(1): 1-3.

Williams, R. S. R., Jara, J. L., Matsufuiji, D. \& Plenge, A. 2011. Trade in Andean condor Vulture gryphus feathers and body parts in the city of Cusco and the Sacred Valley, Cusco region, Peru. Vulture News, 61: 16-26.

Williamson, D. F. 2002. In the Black: Status, management, and trade of the American Black Bear (Ursus americanus) in North America. TRAFFIC North America, World Wildlife Fund. Washington, D. C., USA.

World Society for the Protection of Animals (WSPA). 2002a. Animals International, 65:21-25.

World Society for the Protection of Animals (WSPA). 2002b. The bear bile business: The global trade in bear products from China to Asia and beyond. London, UK.

World Society for the Protection of Animals (WSPA). 2005. Finding herbal alternatives to bear bile. Seattle, USA. 